\title{
O TRABALHO NA ESTRATÉGIA SAÚDE DA FAMÍLIA E A PERSISTÊNCIA DAS PRÁTICAS CURATIVISTAS
}

\author{
THE WORK IN THE FAMILY HEALTH STRATEGY AND \\ THE PERSISTENCE OF CURATIVE PRACTICES
}

\author{
EL TRABAJO EN LA ESTRATEGIA SALUD DE LA FAMILIA Y LA \\ PERSISTENCIA DE LAS PRÁCTICAS CURATIVISTAS
}

\author{
Geraldo Eduardo Guedes de Brito ${ }^{1}$ \\ Antonio da Cruz Gouveia Mendes ${ }^{2}$ \\ Pedro Miguel dos Santos Neto ${ }^{3}$
}

Resumo Trata-se de pesquisa com o objetivo de analisar a percepção sobre o trabalho na Estratégia Saúde da Família. Foi realizado um estudo de caso em João Pessoa, no estado da Paraíba, em 2014, que utilizou questionário aplicado a uma amostra de trabalhadores de nível superior $(\mathrm{n}=342)$, entrevistas com gestores $(\mathrm{n}=6)$, profissionais $(\mathrm{n}=12)$ e observação simples in loco. Os dados foram tratados por meio da análise do conteúdo e estatística descritiva. A finalidade do trabalho da Estratégia Saúde da Família mais evidente no componente qualitativo foi de melhorar as condições de saúde dos usuários. Concretizava-se pela prevenção, promoção, educação e tratamento de doenças, incorporando o conceito ampliado de saúde e autonomia da população. Porém, de acordo com os dados quantitativos, era dispensado pouco tempo para tais atividades quando comparado ao destinado à demanda espontânea, gerando um expressivo número de consultas individuais. Práticas de educação, prevenção, promoção e abordagens em grupo devem ser estimuladas e incorporadas ao cotidiano da Estratégia Saúde da Família de maneira transversal a todas as ações, no qual a clínica ampliada é uma potente ferramenta. O maior envolvimento dos profissionais nestas atividades qualificará a assistência por meio da interprofissionalidade e favorecerá o rompimento com as práticas curativistas.

Palavras-chave estratégia saúde da família; trabalho; recursos humanos; estudo de caso.
Abstract The research had the goal of analyzing the perception regarding the work on the Family Health Strategy. We conducted a case study in the city of João Pessoa, in the state of Paraíba, Brazil, in 2014, with a questionnaire applied to a sample of workers with higher education $(\mathrm{n}=342)$, interviews with managers $(\mathrm{n}=6)$, professionals $(\mathrm{n}=12)$, and a simple in loco observation. The data were treated by means of an analysis of the content and by descriptive statistics. The most evident qualitative goal of the work performed by the Family Health Strategy was improving the health conditions of the users. It was put into effect though the prevention, promotion, education and treatment of diseases, incorporating the expanded concept of health and autonomy of the population. However, according to the quantitative data, little time was devoted to these activities when compared to the time devoted to the spontaneous demands, generating a significant number of individual consultations. Activities regarding education, prevention, promotion and group approaches must be stimulated and incorporated into the everyday activities of the Family Health Strategy in a manner that intersects all of the actions, in which the expanded medical practice is a powerful tool. More involvement on the part of the professionals in these activities will enhance the quality of the assistance though interprofessionality, and will help break free from curative practices. Keywords Family health strategy; labor; human resources; case study. 


\section{Introdução}

No contexto da organização do setor público de saúde no Brasil, a Atenção Primária à Saúde ou Atenção Básica $(\mathrm{AB})$, representada preferencialmente pela Estratégia Saúde da Família (ESF), é o principal mecanismo de reorganização do Sistema Único de Saúde (SUS). A Política Nacional de Atenção Básica (PNAB) apresenta o caráter inovador da proposta de práticas no âmbito da ESF. Espera-se que ela provoque impactos positivos, não apenas nas condições de saúde da população, mas também em seus determinantes e condicionantes socioculturais (Brasil, 2012).

De acordo com dados do Departamento de Atenção Básica do Ministério da Saúde, em fevereiro de 2015, 5.296 municípios possuíam a ESF implantada, cobrindo aproximadamente $57 \%$ da população brasileira (Brasil, 1998). Porém, a consolidação da ESF, para além de sua cobertura populacional, ainda enfrenta entraves, relacionados tanto ao seu processo de trabalho quanto ao modelo de produção do cuidado adotado pelos trabalhadores, em especial nas grandes cidades (Nunes et al., 2014).

Os gestores e os trabalhadores do setor saúde são responsáveis pela implantação, implementação, avaliação e execução in loco das políticas públicas de saúde (Cezar-Vaz et al., 2009). Cabe a estes atores incorporarem, no planejamento e organização dos sistemas locais de saúde e em seu processo de trabalho, os princípios organizativos e doutrinários do SUS. Assim, pode-se eliminar o descompasso entre a finalidade do trabalho em saúde prescrito nos dispositivos normativos e o realizado no trabalho vivo em ato.

Para Marx, a finalidade do trabalho ou, como ele define, "o trabalho em si" trata-se da adequação de uma atividade para se alcançar um fim determinado, orientando a intencionalidade do trabalho para atender determinadas necessidades humanas (Marx, 2012). No campo da saúde, em específico, o trabalho se materializa com base na construção conceitual de sua finalidade (Cezar-Vaz et al., 2009), refletindo no planejamento e execução das ações que buscam atender as demandas em saúde da população.

Compreender a concepção dos trabalhadores e gestores municipais sobre a finalidade da ESF pode contribuir para o entendimento da materialização do trabalho neste modelo de atenção à saúde e identificar mudanças no modo de se produzir cuidado neste nível de atenção. Nesse contexto, o objetivo desse artigo foi conhecer a percepção de trabalhadores de nível superior e gestores sobre a finalidade do trabalho na ESF no município de João Pessoa, capital do estado da Paraíba. 


\section{Percurso metodológico}

Trata-se de um estudo de caso, conduzido em João Pessoa, na Paraíba, que utilizou a metodologia qualitativa e quantitativa por meio da aplicação de um questionário autoaplicado a uma amostra de trabalhadores de nível superior da ESF $(n=342)$, realização de entrevistas em profundidade com gestores $(n=6)$ e trabalhadores de nível superior da ESF $(n=12)$ e observação simples in loco. O emprego de métodos e técnicas de pesquisa combinados (triangulação de métodos) não os opõe, pelo contrário, eles se complementam e interagem. Além disso, a triangulação de métodos permite suprir lacunas e tornar mais completa a coleta de informações realizadas em campo (Minayo, 2010).

O componente quantitativo do estudo possui como população-alvo o total de 522 trabalhadores de nível superior (175 cirurgiões-dentistas, 179 enfermeiros e 168 médicos) da ESF do município de João Pessoa em fevereiro de 2014. A amostra mínima $(n=261)$ foi calculada pela fórmula que adota a proporção como parâmetro (Bassab, Miazaki e Andrade, 1990), e estratificada proporcionalmente ao número total de cada categoria profissional de interesse ( cirurgiões-dentista $=87$, enfermeiros $=89$, médicos $=85$ ). Foi estimada uma perda amostral de $40 \%$, pelo resultado anterior de uma pesquisa que usou questionário autoaplicado com trabalhadores da ESF (Zanetti et al.; 2010).

Para a captação dos sujeitos para a malha amostral, foram sorteadas aleatoriamente 140 Unidades de Saúde da Família, obedecendo também a sua distribuição proporcional nos cinco Distritos Sanitários. Os dados foram coletados por meio de um questionário semiestruturado e autoaplicado. Esse instrumento foi baseado na revisão da literatura e continha 47 questões. Dente elas, havia algumas sobre características de identificação (5 questões), de formação (18 questões) e do trabalho na ESF (24 questões).

Foram convidados 366 trabalhadores para participar do estudo e destes, 24 não aceitaram. Definiu-se como critério de inclusão na amostra ser trabalhador de nível superior vinculado a uma eSF (Equipe de Saúde da Família) do município de João Pessoa; e como critério de exclusão, a sua recusa em participar do estudo. As características gerais da amostra do componente quantitativo do estudo foram devidamente apresentadas e discutidas anteriormente por Brito e colaboradores (2016).

Na elaboração dos resultados, foram consideradas as seguintes variáveis de caracterização do trabalho na ESF: percentual médio de tempo semanal dedicado a atividades de prevenção e promoção da saúde; número de ativida- 
des em grupo por semana; número de consultas semanais; percentual médio de tempo semanal dedicado ao atendimento de demanda espontânea. Para a análise dos dados, foi empregada a estatística descritiva, e as variáveis foram estratificadas pelas profissões. Os resultados foram gerados pelo software de domínio público EpiInfo $7^{\mathrm{R}}$.

O componente qualitativo foi composto pela realização de entrevistas em profundidade com seis gestores municipais, 12 trabalhadores de eSFs e três trabalhadores do Núcleo de Apoio à Saúde da Família do município de João Pessoa. A coleta dos dados ocorreu durante o período de agosto à novembro de 2014, individualmente e no ambiente de trabalho dos sujeitos, por um único pesquisador devidamente treinado. As entrevistas foram gravadas, transcritas e submetidas à validação.

Inicialmente, foram realizadas entrevistas individuais com gestores municipais (quatro diretores de Distrito Sanitário, a diretora de $\mathrm{AB}$ e o diretor de Gestão do Trabalho da Secretaria Municipal de Saúde de João Pessoa). O roteiro da entrevista visava apreender as percepções e expectativas institucionais da gestão acerca da finalidade, do objeto e instrumentos de trabalho e do trabalho vivo na ESF. Além disso, aos diretores de Distrito Sanitário foi solicitada a indicação de três eSFs com os seguintes critérios: ser a mesma equipe de médico, enfermeiro e cirurgião-dentista por pelo menos doze meses; que desenvolvessem atividades de atenção individual e coletiva; e que fossem consideradas eSFs de referência de trabalho em seu distrito sanitário.

Posteriormente, entrevistaram-se os profissionais de nível superior de três eSFs indicadas pelos diretores de Distrito Sanitário (três médicos, três enfermeiros e três cirurgiões-dentistas) e de um trabalhador do Núcleo de Apoio à Saúde da Família de cada uma dessas três eSFs. O roteiro da entrevista da segunda fase apreendeu as percepções dos trabalhadores acerca da finalidade, do objeto e instrumentos de trabalho e do trabalho vivo na ESF. Para a definição do tamanho amostral da segunda fase foi adotado o critério de saturação (Fontanella e Magdaleno Júnior, 2012).

Foram efetivamente realizadas 18 entrevistas. Os sujeitos deste componente do estudo foram predominantemente do sexo feminino $(78,0 \%)$. Gestores apresentaram média de idade de 32 anos e trabalhadores de 41 anos. Todos os entrevistados desempenhavam suas funções do momento da entrevista há pelo menos um ano. Duas eSFs deste estudo obtiveram certificação no Programa Nacional de Melhoria do Acesso e da Qualidade da Atenção Básica (PMAQ$\mathrm{AB})$ com avaliação muito acima da média e uma com certificação mediana.

Além das entrevistas, procedeu-se a uma observação simples no local de trabalho, durante o período de agendamento e realização das entrevistas nas USF dos sujeitos, devidamente registrada no diário de campo. Gil (2008) en- 
tende que esse tipo de observação se refere àquela em que o pesquisador observa, de maneira espontânea, os fatos de determinada realidade em um local.

Para a elaboração deste artigo, foi analisada a entrevista em profundidade impulsionada pela pergunta: "Qual a finalidade do seu trabalho na ESF?"4 e "para a gestão qual a finalidade do trabalho na ESF?". ${ }^{5} \mathrm{O}$ tratamento dos dados qualitativos foi realizado por meio da análise do conteúdo, orientada por uma abordagem interpretativa e crítica do material produzido (Bardin, 2012). Os trabalhadores foram identificados pela letra " $T$ ", gestores pela " $G$ " e numerados aleatoriamente.

Todos os sujeitos convidados aceitaram, livremente, participar do estudo e assinaram o termo de consentimento livre e esclarecido. Esta pesquisa foi aprovada (CAEE n. 32415114.8.0000.5190) pelo Comitê de Ética em Pesquisa do Centro de Pesquisas Aggeu Magalhães - Fiocruz/Pernambuco. O estudo contou com o financiamento do Ministério da Ciência, Tecnologia e Inovação e do Conselho Nacional de Desenvolvimento Científico e Tecnológico (CNPq) (Chamada universal - MCTI/CNPq n. 14/2014).

\section{A definição da finalidade}

A finalidade do trabalho da ESF que emergiu mais fortemente na análise do conteúdo das entrevistas em profundidade deste estudo, e que é apresentada e discutida neste artigo, foi a de promover a melhoria das condições de saúde dos usuários adscritos. Esta percepção já era esperada, uma vez que é a finalidade do trabalho em saúde de maneira geral, e não uma característica exclusiva daquele desenvolvido na ESF.

Merhy e colaboradores (2004) asseveram que a finalidade de qualquer trabalho em saúde é o "ato de cuidar". Vasconcelos (2008) define o cuidado como o comprometimento baseado na identificação das necessidades passíveis de ser enfrentadas de acordo com suas exigências. Para Anéas e Ayres (2011), o tema cuidado em saúde tem sido abordado no campo da Saúde Coletiva, com base em duas concepções aparentemente antagônicas: uma baseada na instrumentalidade, centrada em procedimentos e no tecnicismo técnico, e outra, nos aspectos relacionais no encontro entre trabalhadores e usuários dos serviços de saúde.

Os entrevistados avançaram e contextualizaram as melhorias das condições de saúde que extrapolaram a concepção reducionista da saúde como ausência de doença, e incorporaram em suas falas, questões do modelo de determinação social da saúde. Além disso, sugeriram que o cuidado produzido neste nível de atenção deve gerar a autonomia nos usuários em relação a seus problemas de saúde e aos problemas da comunidade onde vivem. 
Melhorar a qualidade de vida das pessoas, melhorar a saúde das pessoas e contribuir não só em relação à saúde, até para abrir os olhos para orientar como melhorar, até ensinar um caminhozinho que você pode chegar lá para descobrir certos atalhos para conseguir melhoras pra seu bairro e sua comunidade de maneira geral (T5).

[Quanto às expectativas do Distrito Sanitário em relação à ESF] A responsabilização pelos usuários que estão adscritos às unidades deles. E aí, essa responsabilização, que eu acho que é a mais fundamental, é em relação a tudo que se dê naquela comunidade. Sejam todas as questões envolvendo a saúde, de se pensar na melhoria de moradia, de educação, porque tudo isso vai influenciar na questão da saúde, ou de até fazer o seu autocuidado (G1).

No Brasil, a $\mathrm{AB}$ se caracteriza por um conjunto de ações (materialização da finalidade do trabalho) individuais e coletivas com a finalidade de oferecer à população atendida uma atenção integral centradas nas necessidades singulares e coletivas de saúde da população adscrita (Brasil, 2012). Cezar-Vaz e colaboradores (2009), ao realizarem uma descrição qualitativa do sistema de significados atribuídos por médicos e enfermeiros da cidade de Pelotas, no Rio Grande do Sul, à finalidade do trabalho na ESF, verificaram que os sujeitos entrevistados a compreendiam como o objetivo de melhorar as condições de saúde e da qualidade de vida, o que também foi verificado por este estudo e é corroborado pela PNAB (Brasil, 2012).

Assim, dois aspectos relevantes emergiram nas falas dos entrevistados no que diz respeito à finalidade do trabalho na ESF: a incorporação do conceito de determinação social da saúde e de geração de autonomia aos usuários. Trata-se de questões que sinalizam fortemente mudança de paradigma de produção do cuidado, que rompe com a concepção instrumental, tecnicista, autoritária e vertical das práticas de saúde historicamente construídas e consolidadas no Brasil.

Cabe destacar que a definição desta finalidade sinaliza um avanço conceitual, uma vez que não restringiu o trabalho em saúde à cura de doenças e sublinha a potência da ESF para impulsionar mudanças de práticas no setor saúde.

\section{A materialização da finalidade: o fazer}

Este estudo identificou, por meio das entrevistas em profundidade com trabalhadores de nível superior e gestores, que, para se alcançar a finalidade de melhorar as condições de saúde da população adscrita, os trabalhadores das eSFs reconhecem que as ações de prevenção e promoção da saúde, educação em saúde e tratamento de doenças instaladas são as mais relevantes 
e presentes em seu cotidiano. A seguir, esses resultados são apresentados e discutidos.

\section{A prevenção e a promoção da saúde}

As ações de prevenção e promoção da saúde foram veementemente indicadas pelos entrevistados deste estudo como as principais formas do trabalho na ESF para melhorar as condições de saúde da população. Este resultado também foi descrito por Cezar-Vaz e colaboradores (2009) e definido pela PNAB como atividades a serem desenvolvidas pelas eSFs (Brasil, 2012).

A ESF assumiu para os entrevistados o acompanhamento dos usuários para além do manejo de doenças já instaladas e inseriu-se no campo da promoção e da prevenção da saúde, ao lançarem mão de estratégias de educação em saúde e reconhecerem a amplitude do escopo dessas atividades, rompendo com o modelo curativista e biologicista, conforme ilustrado pelos fragmentos a seguir.

[A finalidade do trabalho na ESF] é bem amplo mesmo, assim, é bem amplo, mas a finalidade principal é prevenir e promover a saúde das pessoas de forma bem integral (T4).

(...) promover... uma promoção à saúde e uma prevenção pra solucionar o que for aqui na Unidade (T10).

É o desafio que nós assumimos a partir exatamente dessa compreensão da percepção da mudança do modelo assistencial. Hoje o desafio que nós temos em pauta é atuarmos na perspectiva da prevenção e promoção da saúde (G7).

Campos (2003) assevera que a prioridade dada, durante o período de implantação da ESF no Brasil às atividades de prevenção e promoção da saúde, tem uma forte conotação política. Atribui esse fato à necessidade de alterar a trajetória histórica da $\mathrm{AB}$, no Brasil, até então hegemônica, o que justifica a percepção dos entrevistados desta pesquisa.

Porém, os resultados do componente quantitativo (Tabela 1) apontaram que o percentual médio de tempo semanal dedicado pelos trabalhadores de nível superior às atividades de prevenção e promoção da saúde ainda é discreto, uma vez que aproximadamente $60 \%$ da amostra declarou dedicar de zero a $20 \%$ de seu tempo de trabalho semanal na ESF a atividades de prevenção e promoção da saúde. A média deste indicador para amostra foi de 32,5\% do tempo de trabalho/semana e a análise por categoria profissional apresentou resultados semelhantes para cirurgiões-dentistas, médicos e enfermeiros. 
Percentual médio de tempo semanal dedicado a atividades de prevenção e promoção da saúde $(n=342)$, João Pessoa - 2014

\begin{tabular}{lllllllll}
\hline \multirow{2}{*}{ Percentual } & \multicolumn{1}{l}{ Amostra } & \multicolumn{2}{c}{ Enfermeiros } & \multicolumn{2}{c}{ Cirurgiões-dentistas } & \multicolumn{2}{l}{ Médicos } \\
\cline { 2 - 9 } & $\mathrm{n}$ & $\%$ & $\mathrm{n}$ & $\%$ & $\mathrm{n}$ & $\%$ & $\mathrm{n}$ & $\%$ \\
\hline Até 20\% & 205 & 59,9 & 73 & 57,5 & 70 & 59,3 & 62 & 63,9 \\
De 21\% a 50\% & 67 & 19,6 & 30 & 23,6 & 25 & 21,2 & 12 & 12,4 \\
De 51 a 100\% & 70 & 20,5 & 24 & 18,9 & 24 & 19,5 & 23 & 23,7 \\
\hline
\end{tabular}

Fonte: Os autores.

Este discreto percentual de tempo de trabalho semanal dedicado a atividades de prevenção e promoção da saúde, verificado entre os trabalhadores de João Pessoa, pode ser uma consequência da expressiva demanda por atendimento curativista individual para a ESF, conforme sinalizaram os resultados. Horta e colaboradores (2009), ao investigar as atividades de promoção da saúde em Contagem, no estado de Minas Gerais, concluíram que as dificuldades enfrentadas pelos trabalhadores para efetivarem essa prática se relacionavam a uma importante demanda espontânea associada a uma prática curativista hegemônica nas USF. Apontam, ainda, o número insuficiente de profissionais inseridos nas eSFs, o que gera uma sobrecarga de trabalho. Outro aspecto foi referente à própria resistência dos usuários a participarem de tais atividades.

A Carta de Ottawa (OMS, 1986) define a promoção da saúde como o processo de capacitação da comunidade para atuar na melhoria de sua qualidade de vida e saúde, incluindo mais participação no controle desse processo. Em 2006, foi publicada a Política Nacional de Promoção da Saúde, cujo objetivo geral é o de melhorar a qualidade de vida e reduzir situações de vulnerabilidade e de riscos à saúde associados aos seus determinantes e condicionantes, como, por exemplo, o estilo de vida, as condições de trabalho, moradia, meio ambiente, educação, lazer, cultura e o acesso a bens e serviços essenciais. Logo, trata-se de uma estratégia de articulação, que visa criar ações que diminuam as situações de vulnerabilidade, defendam radicalmente a equidade e incorporem a participação e o controle social (Brasil, 2010).

Para alcançar o conceito ampliado de saúde, os indivíduos e os grupos devem ser capazes de identificar seus desejos, satisfazer às suas necessidades e modificar positivamente o meio ambiente. Nessa definição, a saúde assume uma conotação positiva e que enfatiza os recursos sociais e pessoais, bem como as capacidades físicas. Em síntese, a promoção da saúde, de acordo com esse documento, não é responsabilidade exclusiva do setor de saúde e vai além de um estilo de vida saudável, na direção de um bem-estar global. Czeresnia (2003) afirma que a promoção da saúde, 
em suas múltiplas dimensões, não pode ser delegada exclusivamente a uma área de conhecimento, o que reforça seu caráter intersetorial.

As atividades de prevenção são aquelas cuja intenção é de evitar o aparecimento e o estabelecimento de acometimentos à saúde. Assim, buscam reduzir a incidência e a prevalência de determinadas doenças, baseando-se, predominantemente, no conhecimento epidemiológico moderno (Czeresnia, 2003), que é construído com base na história natural da doença e intervém em diversos fatores de risco para o aparecimento de doenças. Atualmente, emprega-se uma classificação em quatro níveis para a prevenção.

A mais conhecida recentemente - a 'prevenção primordial' - recomenda que se deve evitar o surgimento e a consolidação de padrões de vida social, econômicos e culturais que aumentam o risco de adoecer; a 'prevenção primária' objetiva controlar a incidência de agravos mediante intervenções em suas causas e fatores de risco; já a 'prevenção secundária' relaciona-se com a cura e o acompanhamento de casos de doenças já instaladas, reduzindo as sequelas por meio do diagnóstico e do tratamento precoce dos usuários, e a 'prevenção terciária', cujo objeto de intervenção é a redução do progresso e das complicações de uma doença já estabelecida com a aplicação de medidas orientadas para reduzir sequelas e deficiências (OPAS, 2007).

Ao considerarmos o papel da ESF na coordenação do cuidado dos usuários adscritos, a 'prevenção quaternária' emerge como uma importante estratégia produtora de cuidado. De acordo com Bentzen (2003), a prevenção quaternária trata-se da identificação de usuários em risco de tratamento excessivo para protegê-los de novas intervenções clínicas desnecessárias e propor alternativas para a abordagem do problema de saúde eticamente aceitáveis. Conforme apontado por Norman e Tesser (2009) na atenção primária à saúde, a prevenção quaternária tem se tornado uma prática constante, representando um contraponto à definição de saúde, fator de risco e doença.

Assim, é de mister importância que os trabalhadores da ESF orientem suas ações de prevenção baseados no conceito de prevenção quaternária. Com isso, poderão reduzir a medicalização excessiva de fatores de risco e também a solicitação de exames clínicos e complementares ao estritamente necessário.

Com base nas conceituações apresentadas, é possível identificar as diferenças entre promoção da saúde e prevenção. Porém, evidências sugerem que existe uma dificuldade de se distinguirem claramente essas estratégias na prática cotidiana (Czeresnia, 2003), o que pode representar uma fragilidade dos trabalhadores da ESF de um modo geral e deve ser considerado para a realidade pessoense. Tesser e colaboradores (2010) identificaram que os integrantes de eSFs de Florianópolis, em Santa Catarina, não definiam adequadamente os dois termos. Grande parte dos profissionais entendia a promoção de saúde como prevenção de doenças. Resultado semelhante foi descrito por Antonia Santos e colaboradores (2012), ao investigarem os sentidos atribuídos às práticas de 
promoção da saúde do adolescente por trabalhadores da ESF, em Fortaleza/ CE, e por Horta et al. (2009), em uma pesquisa qualitativa realizada em Belo Horizonte e em Contagem, Minas Gerais.

Essa confusão conceitual é consequência da inclusão da promoção da saúde no nível de prevenção primária no clássico livro de Medicina Preventiva de Leavell e Clark e das próprias políticas relacionadas ao tema, como o caso da Política Nacional de Promoção da Saúde (Brasil, 2010) e da PNAB (Brasil, 2012). Essas políticas públicas propõem ações de promoção da saúde que podem ser caracterizadas como de prevenção de doenças.

Como estratégia para preencher essa possível lacuna conceitual, sugere-se o investimento em educação permanente e que se definam com mais clareza os termos e as estratégias para suas efetivações, na prática, em documentos orientadores do trabalho em saúde. Para Tahan e Carvalho (2010), além de capacitar adequadamente os trabalhadores, é necessário que eles se comprometam em planejar e implementar essas atividades e considerem sempre os aspectos intersetoriais e multidisciplinares para manejar a complexidade das questões relacionadas ao processo saúde-doença. Moura e colaboradores (2014) apontam que a educação permanente é a principal ferramenta para a capacitação dos trabalhadores da rede de atenção à saúde.

\section{A educação em saúde}

A educação em saúde, que emergiu nas falas dos entrevistados associada às atividades de promoção da saúde e prevenção de doenças, pode ser considerada uma estratégia metodológica de realização dessas atividades. Para Oliveira e Wendhausen (2014), a educação em saúde é uma potente ferramenta, com a qual se pode modificar o modelo assistencial e impactar e transformar positivamente as condições de saúde da população.

A gente tenta muito conscientizar, fazer um trabalho em saúde mesmo, está educando mesmo aquelas pessoas, fazendo a promoção, não só a prevenção, mas a promoção de saúde (T12).

Trabalhar na Saúde da Família pra mim é proporcionar aos pacientes uma assistência de maior qualidade, mas não só a parte assistencial, mas também promover a saúde com atividades, com ações de educação em saúde (T6).

Alves (2005) concebe que a prática educativa em saúde contemporânea deve visar o desenvolvimento da autonomia e da responsabilidade dos indi- 
víduos no cuidado com a saúde. Ela abandona os métodos de transmissão vertical e autoritária de conhecimentos técnicos e científicos pelos trabalhadores da saúde e incorpora trocas interpessoais de diálogo que favorecem o desenvolvimento de uma análise crítica sobre a realidade e o aperfeiçoamento das estratégias de conquistas e enfrentamento positivo dos problemas de saúde.

De acordo com Tahan e Carvalho (2010), a participação de idosos em um grupo de promoção da saúde em uma USF possibilitou para esses usuários um espaço de convivência e de troca de experiências. Segundo relatos dos sujeitos participantes, transformou, de modo significativo, tanto suas relações sociais quanto suas condições de saúde e, consequentemente, a qualidade de sua vida.

Oliveira e Wendhausen (2014), ao investigarem a concepção de educação em saúde de três eSFs do litoral paranaense, constataram que os discursos dos sujeitos revelavam as dificuldades em diferentes aspectos do agir educativo em suas práticas e fortes resquícios de uma prática bancária, preventiva e medicalizada. De acordo com esses autores, as eSFs do município estudado precisavam romper com essa característica de produção do cuidado em atividades educativas verticalizadas e incorporar o diálogo entre trabalhadores e usuários, por meio de um discurso e de uma prática transformadores, com a inserção dos usuários em todo o processo. Concluem que não basta implantar a ESF, mas também investir em formação e capacitação de gestores e trabalhadores da saúde, visando ao estímulo da adoção de práticas reflexivas e dialogadas.

Com base em uma revisão sistemática da literatura, Junqueira e Santos (2013) verificaram que as atividades de educação em saúde, na ESF, eram efetivadas, majoritariamente, por meio de grupos e palestras. De acordo com Escorel e colaboradores (2007), elas são novas práticas assistenciais incorporadas pela ESF e foram desenvolvidas em proporções semelhantes em seu estudo por médicos e enfermeiros, diferenciando-se do verificado em João Pessoa.

Os resultados quantitativos novamente sinalizaram uma inconsistência entre o declarado nas entrevistas em profundidade e a amostra de trabalhadores de nível superior no que se refere à inserção dos trabalhadores de nível superior em atividades de grupo (Tabela 2). Foi verificada, para a amostra, a participação em uma média de 1,3 atividades de grupo $(\mathrm{dp}=0,9)$ por semana, com um maior envolvimento de enfermeiros e cirurgiões-dentistas quando comparados aos médicos. 
Tabela 2

\begin{tabular}{|c|c|c|c|c|c|c|c|c|}
\hline \multirow{2}{*}{ Número de atividades em grupo/semana } & \multicolumn{2}{|c|}{ Amostra } & \multicolumn{2}{|c|}{ Enfermeiros } & \multicolumn{2}{|c|}{ Cirurgiões-dentistas } & \multicolumn{2}{|c|}{ Médicos } \\
\hline & $\mathrm{n}$ & $\%$ & $\mathrm{n}$ & $\%$ & $\mathrm{n}$ & $\%$ & $\mathrm{n}$ & $\%$ \\
\hline 0 & 33 & 9,7 & 12 & 9,5 & 8 & 6,8 & 13 & 13,4 \\
\hline 1 & 199 & 58,2 & 73 & 57,5 & 64 & 54,2 & 62 & 63,9 \\
\hline 2 ou mais & 110 & 32,1 & 41 & 33,1 & 46 & 39,0 & 17 & 22,7 \\
\hline
\end{tabular}

Fonte: Os autores

O menor envolvimento dos médicos em atividades de grupo na ESF verificadas neste estudo pode ser uma consequência da visão biologicista com ênfase no atendimento individual e curativo adotada por parte desses trabalhadores. Quando as atividades em grupo e educativas são ofertadas à população sob essa concepção, tendem a ser centradas em relações autoritárias de prescrição de tratamentos, condutas e mudança de comportamentos por meio da transmissão vertical de conhecimentos (Alves e Aerts, 2011).

Durante a observação simples in loco, foi possível identificar que em duas eSFs consideradas de referência por gestores municipais, as ações em grupo de prevenção, promoção e educação em saúde aconteciam facilitadas por atividades de ensino desenvolvidas sem a participação dos trabalhadores da equipe de saúde. Este resultado sugere uma fragilidade no processo de articulação ensino-serviço, uma vez que, quando não incorporadas pelo cotidiano de trabalho da ESF, tornam-se pontuais e não dialógicas entre a academia e as eSFs.

De acordo com Vieira e colaboradores (2016), a integração ensino-serviço deve ser capaz de favorecer o desenvolvimento dos docentes, estudantes e trabalhadores dos serviços de saúde. Brehmer e Ramos (2014) apontam que, ao receberem as atividades de ensino, os serviços de saúde reorganizam seu processo trabalho e que para isso é necessária uma mobilização bilateral. Discutem ainda que, pelo observado no presente estudo, a integração ensino-serviços pode acontecer considerando apenas as necessidades das instituições de ensino, o que compromete a potência transformadora desta aproximação entre a formação para setor saúde com os trabalhadores dos serviços e comunidade.

Pereira e Fracolli (2011) investigaram as percepções de trabalhadores da saúde acerca da articulação ensino-serviço na implantação da vigilância em saúde no município de São Paulo. Um de seus resultados apontou que as atividades desenvolvidas eram limitadas às atividades curriculares, com a participação passiva dos trabalhadores dos serviços. Concluem que, para que a articulação ensino-serviço alcance a transformação do processo de trabalho, é necessária a mudança no estabelecimento das relações e, consequentemente, na tomada de decisões durante as atividades de ensino nos serviços, envolvendo instituições de ensino, trabalhadores, gestores e usuários. 


\section{O tratamento de doenças instaladas}

Além da prevenção e da promoção da saúde, os entrevistados apontaram ainda o papel fundamental da ESF no tratamento de doenças já instaladas. Essa realidade indica uma importante demanda por assistência curativista, o que pode fragilizar a efetivação das atividades de prevenção, promoção e proteção da saúde e contribuir para perpetuar a representação social da função da ESF como um serviço de pronto-atendimento ou ambulatorial.

É onde a gente poderia estar trabalhando para prevenir essas doenças, e ter essa parte curativa, mas é nesse contexto. Hoje está um pouquinho desvirtuado porque chega muita doença (T3).

O povo é cura, cura, cura, né? Remédio, remédio, remédio, mas eu gostaria muito de promover saúde (T1).

Embora as atividades de cura e reabilitação, no âmbito da $\mathrm{AB}$, sejam previstas na PNAB (Brasil, 2012) e, indiscutivelmente, necessárias e inerentes ao trabalho em saúde, a predominância dessas práticas nesse nível de atenção deve servir de sinal de alerta para a possível manutenção do modelo curativista. Assim, a concepção curativista ainda parece estar presente tanto na operacionalização da rede de atenção à saúde quanto no imaginário popular, como a resposta esperada dos serviços de saúde para os problemas de saúde da população.

De acordo com Fortuna e colaboradores (2012, p. 658), evidências na literatura especializada apontam que "a reprodução na ESF do modelo de atenção hegemônico, centrado em ações prescritivas e curativas, é um risco e uma possibilidade iminente". Mesmo reconhecendo os avanços alcançados com o SUS, ainda é possível perceber a persistência da dicotomia entre uma produção de cuidado curativa e uma de prevenção e promoção da saúde (Limongi, Menezes e Menezes, 2009).

Caetano e Dain (2002) discutem, à luz da perspectiva de implantação da ESF em grandes cidades brasileiras, que as regiões metropolitanas são marcadas por conflitos no setor da saúde. Para esses autores, nos centros urbanos, emergem questões como as desigualdades entre a oferta de serviços e as demandas assistenciais e entre os valores profissionais e as necessidades de saúde da população, além de tensões entre práticas em saúde curativistas e a produção do cuidado integral. Adriano Santos e colaboradores (2012), ao analisarem as práticas assistenciais de trabalhadores médicos e enfermeiros da ESF nas capitais Vitória, Florianópolis, Aracaju e Belo Horizonte, verificaram 
que a maior parte das atividades rotineiras desenvolvidas nesses municípios eram majoritariamente as ações individuais voltadas para grupos específicos e a demanda espontânea. Já as atividades de educação em saúde foram apontadas como realizadas diariamente por menos de um terço dos entrevistados.

Essa questão, de acordo com os resultados desta pesquisa, refletiu-se no processo de trabalho das eSFs, na verificação da expressiva demanda por assistência curativista, gerando a necessidade de realização de consultas individuais. O número de consultas realizadas é um indicador que reflete a capacidade da ESF em oferecer assistência individual.

A PNAB (Brasil, 2012) prevê como atribuição dos membros das eSFs a realização de consultas clínicas de acompanhamento a grupos específicos e a demanda espontânea emergencial e de urgência, o que foi declarado pelos entrevistados das três categorias profissionais. Logo, a ESF representa, em João Pessoa, uma importante possibilidade de acesso a cuidados individuais multidisciplinares de saúde, contrapondo-se à assistência médico-centrada.

A equipe vai fazer o acolhimento. E, dependendo da necessidade daquele paciente, ou ele vai pra o enfermeiro ou ele vai pro médico, vai pro dentista ou vai pra os técnicos em enfermagem (T11).

Os resultados quantitativos (Tabela 3) confirmaram a realização de consultas individuais pelas três categoriais profissionais de nível superior da ESF. Os médicos declararam realizar uma média de 115,3 (dp =47,6), enfermeiros $70,0(\mathrm{dp}=32,2)$ e cirurgiões-dentistas $49,1(\mathrm{dp}=21,1)$ consultas semanais. O número médio de consultas realizadas semanalmente foi reproduzido na variável percentual médio de tempo dedicado ao atendimento da demanda espontânea, como esperado. Logo, foi maior entre médicos, seguidos dos cirurgiões-dentistas, quando comparados aos enfermeiros.

\section{Tabela 3}

Número de consultas semanais e percentual médio de tempo dedicado ao atendimento da demanda espontânea (n=342), João Pessoa, 2014

\begin{tabular}{|c|c|c|c|c|c|c|c|c|}
\hline & \multicolumn{2}{|c|}{ Amostra } & \multicolumn{2}{|c|}{ Enfermeiros } & \multicolumn{2}{|c|}{ Cirurgiões-dentista } & \multicolumn{2}{|c|}{ Médicos } \\
\hline & $\mathbf{n}$ & $\%$ & $\mathbf{n}$ & $\%$ & n & $\%$ & $\mathbf{n}$ & $\%$ \\
\hline \multicolumn{9}{|c|}{ Número de consultas semanais } \\
\hline Até 50 & 116 & 33,9 & 42 & 33,1 & 71 & 60,2 & 3 & 3,0 \\
\hline De 51 a 100 & 163 & 47,7 & 69 & 57,3 & 47 & 39,8 & 47 & 48,5 \\
\hline Mais de 100 & 63 & 18,4 & 16 & 12,6 & 0 & 0,0 & 47 & 48,5 \\
\hline \multicolumn{9}{|c|}{$\begin{array}{l}\text { Percentual médio de tempo } \\
\text { semanal dedicado ao atendimento } \\
\text { de demanda espontânea }\end{array}$} \\
\hline Até $25 \%$ & 51 & 14,9 & 25 & 19,7 & 19 & 16,1 & 7 & 7,2 \\
\hline De $26 \%$ a $50 \%$ & 83 & 24,3 & 46 & 36,2 & 19 & 16,1 & 18 & 18,6 \\
\hline De 51 a $100 \%$ & 208 & 60,8 & 56 & 24,1 & 80 & 67,8 & 72 & 74,2 \\
\hline
\end{tabular}

Fonte: Os autores. 
Os resultados das diferenças dessas duas variáveis entre as categorias profissionais podem sinalizar a persistência de traços do modelo hospitalocêntrico em diferentes graus entre essas categorias, já que elas declararam que despendem parcelas de tempo importantes para o atendimento dessa clientela por meio de consultas individuais. Conforme apontam Pimentel e colaboradores (2012), esse modelo de acesso aos serviços de saúde, em que o usuário os procura quando já se encontra com a doença instalada, é hegemônico historicamente e se opõe à lógica assistencial da ESF. Essa questão emergiu na análise das entrevistas como uma questão cultural da população, que representa uma fragilidade para efetivar a capacidade resolutiva da ESF.

Ainda tem muita busca do atendimento médico - mesmo fazendo essa conversa, ainda tem muita busca do atendimento médico (T9).

- Diga moço, o que o senhor deseja?

- Eu quero minha receita.

- Receita de quê?

- Não, eu quero minha receita, olhe, porque eu sou diabético e eu quero minha receita.

- Certo, mas não é assim não senhor, eu preciso conversar, eu não lhe conheço.

- Ah, mas eu não quero fazer consulta não.

- O senhor não quer fazer consulta não?

- Quero não, eu só quero a minha receita.

Aí eu disse:

- Pois o senhor vai precisar aguardar.

A cultura é de antigamente ainda. O que é que eu digo, que quando foi implantado o PSF, as pessoas não foram preparadas (T1).

Porém, da análise do conteúdo das entrevistas em profundidade, emergiu uma percepção de ampliação da abrangência as abordagens individuais no âmbito da ESF. Essa ampliação do olhar, durante as consultas, foi apontada pelos trabalhadores ao reconhecerem que é preciso despender um tempo importante para a sua execução. Essa questão também foi verificada pela observação in loco e registrada no diário de campo (durante a espera para a realização de uma entrevista com um profissional médico, uma usuária que aguardava o profissional declarou ao pesquisador: "A consulta aqui demora, ela quer saber de tudo").

Mas que a gente tenha um algo a mais [além da medicalização]. - Ah doutora, mas a senhora demora muito na sua consulta, ave Maria! O que que vocês estavam fazendo lá esse tempo todinho? Tava só conversando? (T1). 
Aqui é diferente, não adianta eu só trabalhar uma pulpite se eu não ver o contexto social, familiar, então, esse cuidado é mais ampliado. Às vezes vem com aquilo porque é o cuidador que não tem tempo de cuidar porque essa cuidando de outra pessoa (T3)

É necessário considerar que a prática clínica na ESF tem particularidades e deve se diferenciar dos formatos tradicionais da atenção ambulatorial e hospitalar e incorporar novas formas de organizar e registrar em seu processo de trabalho, centrando-se nas necessidades das pessoas contextualizadas à família e à comunidade (Demarzo, Oliveira e Gonçalves, 2011). Saparolli e Adami (2007) apresentam que a prática das consultas individuais, no contexto da ESF, quando realizada de maneira integrada entre os trabalhadores da equipe, possibilitam um diagnóstico ampliado das necessidades de saúde. Isso favorece a adoção de intervenções resolutivas e qualificadas e fortalece o trabalho em equipe, tanto por meio da organização de agendas em comum quanto pela discussão de casos, o que foi apontado pelos entrevistados deste estudo.

Porém, a elevada parcela de tempo dedicada às consultas individuais pelos trabalhadores entrevistados merece ser refletida com certo grau de preocupação. Talvez a ESF esteja assumindo a responsabilidade pelo acompanhamento clínico de casos que deveriam estar nos centros de referência. Porém, sabe-se que a ampliação do acesso aos serviços de saúde propiciado pela expansão e consolidação da ESF não foi acompanhada pela reorganização dos demais níveis de atenção à saúde, em especial, do nível secundário. Conforme apontado por Sousa e colaboradores (2014), o descompasso entre a oferta e as demandas para os serviços de saúde é um fator que favorece a ampliação das iniquidades em saúde. Além disso, o acesso restrito e fragmentado aos serviços especializados pode revelar uma incipiente configuração de rede de atenção à saúde.

Ao verificarmos que as ações de prevenção, promoção e educação em saúde e tratamento de doenças instaladas são realizadas de maneira isolada no cotidiano das eSFs, torna-se necessário sugerir estratégias para superação dessa dicotomia. Indiscutivelmente, essas ações deveriam ser concomitantes e transversais a todas as práticas dos serviços de saúde. Essa integração poderia ser alcançada por meio da clínica ampliada, uma das diretrizes da Política Nacional de Humanização, que se baseia na valorização da escuta qualificada e na ampliação da compreensão do processo saúde-doença.

A contradição verificada entre o declarado nos questionários e nas entrevistas em profundidade pode sinalizar que, embora os trabalhadores tenham uma construção conceitual acerca das práticas na ESF, a gestão e organização do processo do trabalho neste nível de atenção à saúde ainda favoreça a execução de práticas curativistas, baseadas em protocolos, metas e indicadores. Assim, a incorporação da clínica ampliada, conforme proposto por Campos 
e Amaral (2007, p. 852): “além de buscar a produção de saúde, por distintos meios - curativos, preventivos, de reabilitação ou com cuidados paliativos -, a clínica poderá também contribuir para a ampliação do grau de autonomia dos usuários".

\section{Considerações finais}

De acordo com os resultados deste estudo, melhorar as condições de saúde da população adscrita se concretiza no âmbito das eSFs estudadas por meio de ações de prevenção e promoção da saúde, educação em saúde e tratamento das doenças instaladas. Embora as ações de prevenção, promoção e educação em saúde tenham sido bastante indicadas pelos entrevistados, os resultados quantitativos apontaram que os trabalhadores de nível superior ainda dispensam pouco tempo de sua jornada de trabalho semanal para tais atividades. Em contrapartida, foi verificado que a maior parte do tempo da agenda de trabalho dos sujeitos deste estudo era dedicada ao atendimento da demanda espontânea, que gerou, consequentemente, um expressivo número de consultas semanais.

Com essas evidências é possível destacar a necessidade de qualificação da graduação da área de saúde e da educação permanente e no investimento na manutenção e aumento do número de vagas em programas de residência em saúde da família. Com isso, tanto serão formados novos trabalhadores quanto capacitados os já inseridos na rede de atenção à saúde, alinhando-os com os princípios e diretrizes do SUS e, consequentemente, qualificando o trabalho na ESF. Com isso, fragilidades encontradas neste estudo poderão ser superadas.

As práticas de educação, prevenção, promoção e abordagens em grupo devem ser estimuladas e incorporadas ao cotidiano das eSFs. O maior envolvimento dos diversos profissionais nestas atividades poderá qualificar a assistência prestada à população por meio da abordagem interprofissional e também favorecer o rompimento com as práticas médico-centradas nos serviços de saúde. Cabe destacar que esta questão deve ser trabalhada tanto para os profissionais já em serviço quanto durante a graduação para a área de saúde.

Com base na compreensão de que as mudanças no modelo assistencial não podem ocorrer de maneira isolada, fragmentada e por ser de responsabilidade exclusiva da ESF, torna-se fundamental que toda a rede de assistência à saúde seja repensada. Cabe ainda destacar a necessidade da realização de novos estudos que contemplem os trabalhadores não incluídos neste estudo. A ESF se insere em universo complexo, marcado por diversas características objetivas e subjetivas; e ampliar o entendimento do processo de trabalho na ESF por meio da percepção de outros trabalhadores e com outras abordagens metodológicas e de seleção de amostra em muito poderá contribuir para o entendimento do objeto desse estudo. 


\section{Colaboradores}

Geraldo Eduardo Guedes de Brito foi responsável pela concepção, delineamento e redação do artigo. Antonio da Cruz Gouveia Mendes e Pedro Miguel dos Santos Neto orientaram a pesquisa e fizerem a revisão crítica do manuscrito. Não há conflito de interesses.

Resumen Se trata de una investigación con el objetivo de analizar la percepción sobre el trabajo en la Estrategia de Salud de la Familia. Se realizó un estudio de caso en João Pessoa, estado de Paraíba, en el 2014, llevado a cabo a través de un cuestionario aplicado a una muestra de trabajadores de nivel superior $(n=342)$, entrevistas con gestores $(n=6)$, profesionales $(n=12)$ y observación simple in situ. Los datos se trataron por medio del análisis de contenido y estadística descriptiva. La finalidad del trabajo de la Estrategia de Salud de la Familia más evidente en el componente cualitativo fue la de mejorar las condiciones de salud de los usuarios. Se concretaba a través de la prevención, promoción, educación y tratamiento de enfermedades, incorporando el concepto ampliado de salud y autonomía de la población. Sin embargo, de acuerdo con los datos cuantitativos, se dedicaba poco tiempo para dichas actividades en comparación con el destinado a la demanda espontánea, generando un expresivo número de consultas individuales. Las prácticas de educación, prevención, promoción y abordajes en grupo deben estimularse e incorporarse a las tareas cotidianas de la Estrategia de Salud de la Familia de forma transversal a todas las acciones, siendo el componente clínica ampliada una potente herramienta en este sentido. La mayor participación de los profesionales en estas actividades dará más cualificación a la asistencia por medio de la interprofesionalidad y favorecerá la ruptura con las prácticas curativistas.

Palabras clave estrategia de salud de la familia; trabajo; recursos humanos; estudio de caso.

\section{Notas}

${ }^{1}$ Universidade Federal da Paraíba, Centro de Ciências da Saúde, Departamento de Fisioterapia, João Pessoa, Paraíba, Brasil.

$<$ eduardo.guedes.ufpb@gmail.com>

Correspondência: Universidade Federal da Paraíba, Centro de Ciências da Saúde, Campus I, Departamento de Fisioterapia, Cidade Universitária, CEP 56034-360, João Pessoa, Paraíba, Brasil.

${ }^{2}$ Fundação Oswaldo Cruz, Centro de Pesquisas Aggeu Magalhães, Recife, Pernambuco, Brasil. $<$ antoniodacruz@gmail.com>

${ }^{3}$ Fundação Oswaldo Cruz, Centro de Pesquisas Aggeu Magalhães, Recife, Pernambuco, Brasil. <pedromiguel@cpqam.fiocruz.br>

${ }^{4}$ Pergunta feita a trabalhadores.

${ }^{5}$ Pergunta feita a gestores. 


\section{Referências}

ALVES, Gehysa G.; AERTS, Denise. As práticas educativas em saúde e a Estratégia Saúde da Família. Ciência \& Saúde Coletiva, Rio de Janeiro, v. 16, n. 1, p. 319-325, 2011.

ALVES, Vânia S. A health education model for the Family Health Program: towards comprehensive health care and model reorientation. Interface: Comunicação, Saúde, Educação, Botucatu, v. 9, n. 16, p. 39-52, 2005.

ANÉAS, Tatiana V.; AYRES, José R. C. M. Significados e sentidos das práticas de saúde: a ontologia fundamental e a reconstrução do cuidado em saúde. Interface: Comunicação, Saúde, Educação, Botucatu, v. 15, n. 38, p. 651-662, 2011.

BARDIN, Laurence. Análise do conteúdo. São Paulo: Edições 70, 2012.

BASSAB, Wilton O.; MIAZAKI, Édina S.; ANDRADE, Dalton F. Introdução à Análise de Agrupamento. In: SIMPÓSIO NACIONAL DE PROBABILIDADE E ESTATÍSTICA, 9. 1990, São Paulo. Anais..., São Paulo, 1990.

BENTZEN, Niels. Wonca dictionary of general/ family practice. Copenhagen: Maanedskift Lager, 2003.

BRASIL. Ministério da Saúde. Secretaria de Vigilância à Saúde. Secretaria de Atenção à Saúde. Diretrizes Nacionais da Vigilância em Saúde. Brasília, DF: Ministério da Saúde, 2010. Disponível em: <http://livroaberto.ibict.br/ bitstream/1/901/1/diretrizes_nacionais_vigilancia_saude.pdf $>$. Acesso em: 10 nov. 2016.

BRASIL. Ministério da Saúde. Política Nacional de Atenção Básica. Brasília, DF, 2012. Disponível em: <http://189.28.128.100/dab/docs/ publicacoes/geral/pnab.pdf $>$. Acesso em: 10 nov. 2016.

BRASIL. Ministério da Saúde. Sistema de Informação da Atenção Básica. 1998. <http://
www2.datasus.gov.br/SIAB/index.php > . Acesso em: 27 ago. 2015.

BREHMER, Laura C. F.; RAMOS, Flávia R. S. Experiências de integração ensino-serviço no processo de formação profissional em saúde: revisão integrativa. Revista Eletrônica de Enfermagem, Goiânia, v. 16, n. 1, p. 228-237, 2014.

BRITO, Geraldo E. G.; et al. Perfil dos trabalhadores da Estratégia Saúde da Família de uma capital do nordeste do Brasil. Revista de APS, Juiz de Fora, v.19, n.3, p.434-445, 2016.

CAETANO, Rosângela; DAIN, Sulamis. O Programa de Saúde da Família e a reestruturação da atenção básica à saúde nos grandes centros urbanos: velhos problemas, novos desafios. Physis: Revista de Saúde Coletiva, Rio de Janeiro, v. 12, n. 1, p. 11-21, 2002.

CAMPOS, Carlos E. A. O desafio da integralidade segundo as perspectivas da vigilância da saúde e da saúde da família. Ciência \& Saúde Coletiva, Rio de Janeiro, v. 8, n. 2, p. 569-584, 2003.

CAMPOS, Gastão W. S. AMARAL, Márcia A. A clínica ampliada e compartilhada, a gestão democrática e redes de atenção como referenciais teórico-operacionais para a reforma do hospital. Ciência \& Saúde Coletiva, Rio de Janeiro, v. 12, n. 4, p. 849-859, 2007.

CEZAR-VAZ, Marta R. et al. Sistema de significados sobre a finalidade do trabalho na Saúde da Família: uma abordagem qualitativa. Revista da Escola de Enfermagem da USP, São Paulo, v. 43, n. 4, p. 915-922, 2009.

CZERESNIA, Dina. Conceito de saúde e a diferença entre prevenção e promoção. In: CZERESNIA, Dina; FREITAS, Carlos M. (org.). Promoção da saúde: conceitos, reflexões, tendências. Rio de Janeiro: Editora Fiocruz, 2003. p. 39-53.

DEMARZO, Marcelo M. P.; OLIVEIRA, Cristina A.; GONÇALVES, Daniel A. Prática clínica 
na Estratégia Saúde da Família: organização e registro. Material institucional UNA-SUS; Unifesp, 2011. Disponível em: <http://www. unasus.unifesp.br/biblioteca_virtual/esf/2/unidades_conteudos/unidade $15 \mathrm{~m} /$ unidade $15 \mathrm{~m}$. pdf $>$. Acesso em: 10 nov. 2016.

ESCOREL, Sarah et al. O Programa de Saúde da Família e a construção de um novo modelo para a atenção básica no Brasil. Revista Panamericana de Saúde Pública, Washington, v. 21, n. 2/3, p. 164-176, 2007.

FONTANELLA, Bruno J. B.; MAGDALENO JÚNIOR, Ronis. Saturação teórica em pesquisas qualitativas: contribuições psicanalíticas. Psicologia em Estudo, Maringá, v. 17, n. 1, p. 63-71, 2012.

FORTUNA, Cinara M. et al. Notas cartográficas do trabalho na Estratégia Saúde da Família: relações entre trabalhadores e população. Revista da Escola de Enfermagem da USP, São Paulo, v. 46, n. 3, p. 657-664, 2012.

GIL, Antonio C. Métodos e técnicas de pesquisa social. 6. ed. São Paulo: Atlas, 2008.

HORTA, Natália C. et al. A prática das equipes de Saúde da Família: desafios para a promoção de saúde. Revista Brasileira de Enfermagem, Brasília, v. 62, n. 4, p. 524-529, 2009.

JUNQUEIRA, Marcelle A. B.; SANTOS, Fabiana C. S. A educação em saúde na Estratégia Saúde da Família sob a perspectiva do enfermeiro: uma revisão de literatura. Revista de Educação Popular, Uberlândia, v. 12, n. 1, p. 66-80, 2013.

LIMONGI, Jean E.; MENEZES, Eyre C.; MENEZES, Antônio C. Vigilância em saúde no Programa Saúde da Família. Hygeia, Uberlândia, v. 4, n. 7, p. 35-44, 2009.

MARX, K. O capital: edição condensada. 3. ed. São Paulo: Edipro, 2012.

MERHY, Emerson E. et. al. (org.). O trabalho em saúde: olhando e experienciando o SUS no cotidiano. 2. ed. São Paulo: Hucitec, 2004.
MINAYO, Maria C. S. Introdução. In: MINAYO, Maria C. S.; ASSIS, Simone G.; SOUZA, Edinilza R. (org.). Avaliação por triangulação de métodos: abordagem de programas sociais. Rio de Janeiro: Editora Fiocruz, 2010. p. 19-51.

NORMAN, Armando H. TESSER, Charles D. Prevenção quaternária na atenção primária à saúde: uma necessidade do Sistema Único de Saúde. Cadernos de Saúde Pública, Rio de Janeiro, v. 25, n. 9, p. 2012-2020, 2009.

NUNES, Altacílio P. et al. Qualidade da Estratégia Saúde da Família: comparação do desempenho de municípios de pequeno e grande porte. Saúde em Debate, Rio de Janeiro, v. 38, n. 102, p. 452-467, 2014.

OLIVEIRA, Silvia R. G.; WENDHAUSEN, Agueda L. P. (Re)significando a educação em saúde: dificuldades e possibilidades da Estratégia Saúde da Família. Trabalho, Educação e Saúde, Rio de Janeiro, v. 12, n. 1, p. 129-147, 2014.

ORGANIZAÇÃO MUNDIAL DA SAÚDE (OMS). Carta de Ottawa para la Promoción de la Salud. Ottawa: OMS, 1986. Disponível em:< https:// www.paho.org >. Acesso em: 11 maio 2018.

ORGANIZAÇÃO PANAMERICANA DE SAÚDE. Estratégia e plano de ação regional para um enfoque integrado à prevenção e controle das doenças crônicas. Washington: PAHO, 2007. Disponível em: <http://www.bvsde.paho. org/texcom/nutricion/reg-strat-cncdspt.pdf > . Acesso em: 11 maio 2018.

PEREIRA, Juliana G.; FRACOLLI, Lislaine A. Articulação ensino-serviço e vigilância da saúde: a percepção de trabalhadores de saúde de um distrito escola. Trabalho, Educação e Saúde, Rio de Janeiro, v. 9, n. 1, p. 63-75, 2011.

PIMENTEL, Fernando C. et al. Caracterização do processo de trabalho das equipes de saúde bucal em municípios de Pernambuco, Brasil, segundo porte populacional: da articulação comunitária à organização do atendimento 
clínico. Cadernos de Saúde Pública, Rio de Janeiro, v. 28, supl. 5, s146-s157, 2012.

SANTOS, Adriano M. et al. Práticas assistenciais das equipes de Saúde da Família em quatro grandes centros urbanos. Ciência \& Saúde Coletiva, Rio de Janeiro, v.17, n. 10, p. 2687-2702, 2012.

SANTOS, Antonia A. G. et al. Sentidos atribuídos por profissionais à promoção da saúde do adolescente. Ciência \& Saúde Coletiva, Rio de Janeiro, v. 17, n. 5, p. 1275-1284, 2012.

SAPAROLLI, Eliana C. L. ADAMI, Nilce P. Avaliação da qualidade da consulta de enfermagem à criança no Programa de Saúde da Família. Acta Paulista de Enfermagem, São Paulo, v. 20, n. 1, p. 55-61, 2007.

SOUSA, Fabiana O. S. et al. Do normativo à realidade do Sistema Único de Saúde: revelando barreiras de acesso na rede de cuidados assistenciais. Ciência \& Saúde Coletiva, Rio de Janeiro, v. 19, n. 4, p. 1283-1293, 2014.

TAHAN, Jennifer; CARVALHO, Antonio C. D. Reflexões de idosos participantes de grupos de promoção de saúde acerca do envelhecimento e da qualidade de vida. Saúde e Sociedade, São Paulo, v. 19, n. 4, p. 878-888, 2010.

TESSER, Charles D. et al. Concepções de promoção da saúde que permeiam o ideário de equipes da Estratégia Saúde da Família da grande Florianópolis. Revista de Saúde Pública de Santa Catarina, Florianópolis, v. 3, n. 1, p. 42-56, 2010.

VASCONCELOS, Eymard M. Educação popular e a atenção à saúde da família. 4. ed. São Paulo: Hucitec, 2008.

VIEIRA, Leila M. et al. Formação profissional e integração com a rede básica de saúde. Trabalho, Educação e Saúde, Rio de Janeiro, v. 14, n. 1, p. 293-304, 2016.

ZANETTI, Tatiele G. et al. Perfil socioprofissional e formação de profissionais de equipes de Saúde da Família: um estudo de caso. Ciência, Cuidado e Saúde, Maringá, v. 9, n. 3, p. 448-455, 2010.

Recebido em 10/11/2016.

Aprovado em 20/09/2017. 